\title{
The Early Study to Laparoscopic Radical Resection of the Mid Transverse Colon Cancer
}

\author{
Benjing Cheng, Jinjian Xiang*, Arpana Shrestha \\ Department of General Surgery, The First Hospital Affiliated to Yangtze University, Jingzhou, China \\ Email: *xiangjjxiangcy@sina.com
}

How to cite this paper: Cheng, B.J., Xiang, J.J. and Shrestha, A. (2019) The Early Study to Laparoscopic Radical Resection of the Mid Transverse Colon Cancer. Yangtze Medicine, 3, 90-100.

https://doi.org/10.4236/ym.2019.32010

Received: October 10, 2018

Accepted: March 19, 2019

Published: March 22, 2019

Copyright $\odot 2019$ by author(s) and Scientific Research Publishing Inc. This work is licensed under the Creative Commons Attribution International License (CC BY 4.0).

http://creativecommons.org/licenses/by/4.0/

\begin{abstract}
Objective: To explore the safety and the short-term curative effect of the application of assisted laparoscopic radical resection for the mid transverse colon cancer. Methods: A retrospective analysis of 25 cases clinical data of radical resection of mid transverse colon cancer from March 2006 to March 2014 in our hospital. Cases were divided into two groups, laparoscopic group with 12 cases and open group with 13 cases. Intra-operative observation indexes (operation time, bleeding volume, the number of dissected lymph nodes), post-operative recovery indexes (flatus time, liquid food intake time, ambulation time, hospitalization time), post-operative complication (incision site infection, abdominal bleeding, intra-abdominal infection, anastomotic fistula, intestinal obstruction and re-hospitalization within 30 days, mortality) and patient's five-year overall survival were observed in two groups. Results: Operation methods including the principle of complete mesocolic excision (CME), colon dissection along the anatomical plane and safe anastomosis method were applied in Laparoscopic group. Average surgery time of Laparoscopic group was longer than open group, but there was no statistically significant difference between the two groups $(p>0.05)$. Intra-operative blood loss in the Laparoscopic group was obviously lower than open group; the difference was statistically significant $(\mathrm{p}<0.05)$. The number of lymph node dissection in laparoscopic group was higher than open group $(\mathrm{p}<0.05)$. Laparoscopic group in the postoperative recovery index including recovery of bowel function, ambulation time and hospitalization time was shorter than that of the open group after operation $(\mathrm{P}<0.05)$. Conclusion: The use of laparoscopic radical resection for the mid transverse colon cancer resulted in acceptable short term curative effects.
\end{abstract}

\section{Keywords}

Colon Cancer, Clinical Treatment Techniques, Laparoscopy, Mid Transverse Colon Cancer 


\section{Introduction}

Colorectal cancer is the most common malignancy of the gastrointestinal tract. Currently surgery is the only possible way to cure colorectal cancer. Since the first laparoscopic colectomy reported in 1991 by Jacobs and Verdeja, minimally invasive colorectal surgery has become more widespread and acceptable, even for malignant disease [1] [2] [3]. Laparoscopic surgery has the advantages of small incision, less post-operative pain, quick recovery and shorter hospital stay [4]-[10]. Mid transverse colon cancer was never included in randomized controlled trials to assess the efficacy of laparoscopic surgery as curative treatment for colon cancer due to difficulty in deciding the appropriate operative procedure and extent of lymph node dissection, as well as technical difficulties with the laparoscopic identification, ligation, and lymph node dissection around the middle colic vessels [11] [12] [13]. However after nearly 20 years of development, the laparoscopic radical operation in colorectal cancer is a considerable progress, especially in the colon. There had been a lot of multi-center prospective randomized controlled studies showing that the laparoscopic radical surgery in colorectal cancer had the same long-term effect as that with open operation, which has been more and more accepted by the surgeons [14] [15] [16]. In our study, 25 patients underwent surgery for the treatment of the mid transverse colon cancer, of which 12 patient underwent assisted laparoscopic radical resection from March 2006 to March 2014. Thus the aim of this study is to assess the short term curative effects of laparoscopic assisted surgery for resection of mid transverse colon cancer.

\section{Materials and Methods}

\subsection{Clinical Data}

Through retrospective analysis of the clinical data of colorectal cancer from March 2006 to March 2014 in our department (Gastrointestinal Surgery, The First Hospital Affiliated to Yangtze University), a total of 414 patients, including 253 cases of colon cancer, 51 cases of transverse colon cancer, were consecutively admitted for operation. Twenty-five cases with carcinoma in the middle of transverse colon were analyzed. 12 cases underwent assisted laparoscopic radical resection (laparoscopic group), 13 cases underwent conventional open radical resection (open group) (Table 1). Post operative systemic chemotherapy was applied to patients with the visceral peritoneum metastasis and regional lymph node metastasis. Pathologic confirmation, colonoscopy, barium enema, computed tomography (CT), ultrasonography, and chest PA were performed for diagnosis in all patients pre-operatively.

Inclusion criteria in this study: 1) radical resection with carcinoma in the middle of transverse colon, 2) pre-operative diagnosis of malignant neoplasm of transverse colon was clear, 3 ) without distant metastasis through pre-operative examination, 4) $R_{0}$ resection by pathological examination. 
Table 1. The data of patients with radical resection of carcinoma in the middle of transverse colon.

\begin{tabular}{cccc}
\hline Variable & Laparoscopic group $(\mathrm{n}=12)$ & Open group $(\mathrm{n}=13)$ & $P$ value \\
\hline Age & $54 \pm 12$ & $52 \pm 12$ & 0.469 \\
Sex (male/female) & $5 / 7$ & $4 / 9$ & 0.688 \\
Pathological staging (I/II/II) & $3 / 4 / 5$ & $2 / 4 / 7$ & 0.781 \\
$\begin{array}{c}\text { Differentiation of adenocarcinoma } \\
\text { (poorly/moderately/well) }\end{array}$ & $4 / 6 / 2$ & $2 / 5 / 6$ & 0.256 \\
\hline
\end{tabular}

Exclusion criteria in this study: 1) the emergency operation case (acute obstruction, perforation), 2) conversion to open surgery due to bleeding or surrounding organs damage during dissection or adhesion of surrounding tissue.

The human subject research review board of our institution approved our study protocol. Before treatment, informed consent was obtained from each patient.

\subsection{Operation Methods}

Both laparoscopic surgery and open surgery strictly follow the tumor radical principle: 1) the complete mesocolic excision (CME) with central vein ligation (CVL) [17] [18], 2) reconstruction of bowel [19]: If the colonic intestine was long enough, direct anastomosis of bowel without tension was done by end-to-end or end to side anastomosis (end to end anastomosis for loose intestinal loop, Figure 1(a). When colonic intestine is slightly tight after intestinal anastomosis, debonding of flexure duodenojejunalis can relieve the oppression to jejunum (additional debonding of flexure duodenojejunalis for slightly tight intestinal loop, Figure 1(b)). In the more obese patients or intestinal loop is thick and short bowel patients, in order to reduce the tension of anastomosis and the oppression to jejunum, penetration method (colon to be anastomosed through the small mesenteric root) is performed (penetration method for thick and short intestinal loop, Figure 1(c)).

Laparoscopically assisted radical operation:

1) Position of patient: supine, legs apart in order to facilitate the operation. Adjusting the patient position during the operation: reverse trendelenburg position with 20 degree tilt to right side for dissecting hepatic flexure of colon, reverse trendelenburg position with 20 degree tilt to left side for dissecting splenic flexure of colon; reverse trendelenburg position when dissecting transverse colon in the middle.

2) Position of Trocar: using five hole method, put the camera into abdominal cavity through $12 \mathrm{~mm}$ Trocar below the umbilicus, the remaining four holes are respectively placed in the left anterior axillary line $2 \mathrm{~cm}$ below the subcostal region with $12 \mathrm{~mm}$ trocar and left midclavicular line at level of umbilicus with 5 $\mathrm{mm}$ Trocar, the position of right upper and right lower trocar to the corresponding left side, using $5 \mathrm{~mm}$ Trocar. But during surgery the position of trocar 


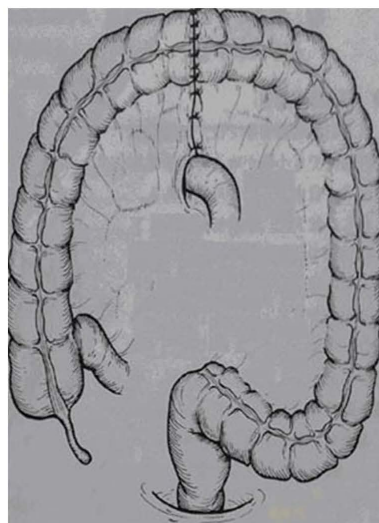

(a)

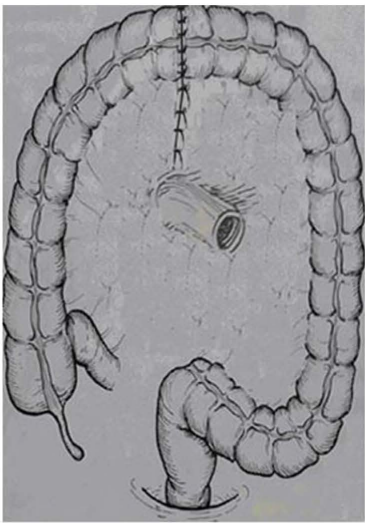

(b)

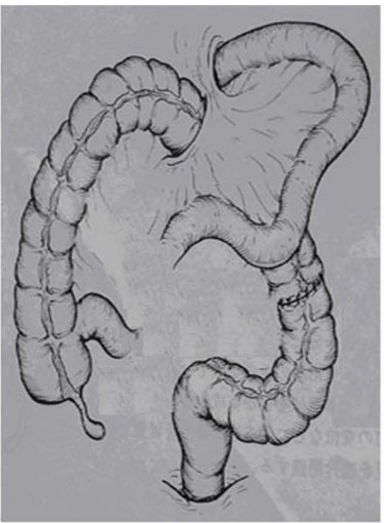

(c)

Figure 1. Reconstruction of bowel.

can be adjusted according to the location of tumor. Pneumoperitoneum is induced with $\mathrm{CO}_{2}$ at $12 \mathrm{mmHg}$.

3) The dissecting process starts with vascular root: After abdominal cavity is explored, greater omentum and transverse colon are pushed to the side of head, small intestine is pushed to the left abdominal cavity, then the mesenteric root is exposed, peritoneal incision is commenced with dissection along the superior mesenteric vein up to the inferior margin of pancreas. Middle colic artery, a branch of superior mesenteric artery, usually found in inferior margin of pancreas is clipped with Ham-Lock clamp and transected at the root along with the middle colic vein. Lymph nodes of the anterior aspect of superior mesenteric vein from middle colic vessels to the gastro-colic trunk of Henle are dissected. A fascial plane between the mesocolon and the underlying retroperitoneum, formed by the fusion of the visceral peritoneum of the mesocolon with the parietal peritoneum of the retroperitoneum is known as Toldt's fascia [20]. During the surgery, dissection along the plane between the intact dorsal mesocolon of the hepatic flexure and the Fredet preduodenopancreatic fascia was done. Then, dissection was done along the duodenal plane into the pancreatic surface, turning left along the pancreatic surface into lesser sac, exposing the tail of the pancreas and the lower pole of the spleen. From the middle of the gastrocolic ligament, besides gastroepiploic vessels arch, greater omentum was dissected towards the hepatic flexure of colon and splenic flexure of colon respectively. Hepatic flexure was mobilized by dissecting the lateral peritoneal fold, the superior attachment (right phrenocolic ligament), and the medial attachment (cholecysto-duodeno-colic ligament). Splenic flexure is mobilized by dissecting the gastrocolic and splenocolic ligament.

4) Resection and Anastomosis: Once the colon was completely mobilized, an incision of about $5-6 \mathrm{~cm}$ was made in the mid line of the upper abdomen. Incision protractor was used. Then the mass was extracted along with the colon. En bloc resection of mass with the omentum and mesentery lymph nodes was done. A stapled colo-colic anastomosis was done with either end-to-end or end-to-side anastomosis. 
5) The colon was then placed back into the abdomen and extraction site was closed. $\mathrm{CO}_{2}$ was reinsufflated to create pneumoperitoneum, operated area was irrigated with saline water. The drainage tube was placed nearby foramen of Winslow. Open operation was done with the same method with middle approach, which was same to laparoscopic group.

\subsection{Observation Indexes}

1) Intra-operative observation indexes: operation time, bleeding volume, the number of dissected lymph nodes.

2) Post-operative recovery indexes: flatus time, liquid food intake time, ambulation time, hospitalization time.

3) Post-operative complication: complication that develops as any adverse event after treatment, including incision site infection, abdominal bleeding, intra-abdominal infection, anastomotic fistula, intestinal obstruction and re-hospitalization within 30 days. Treatment mortality is defined as any death within 30 days of the treatment.

4) Follow-up: Patient's five-year overall survival observed. Post-operative follow-up: every 3 - 6 months for 2 years, then every 6 months for a total of 5 years.

\subsection{Statistical Analysis}

Continuous data were expressed as range and the mean $\pm \mathrm{SD}$ and were compared between groups by using the Independent-Samples T Test. Categorical variables were compared by using the $\chi^{2}$ test. Overall survival was computed by using the Kaplan-Meier method and was compared between the two groups by using the log-rank test. All statistical analyses were performed with the SPSS statistical package (SPSS 20.0 for Windows). A P value $<0.05$ was considered statistically significant.

\section{Results}

\subsection{Patient Characteristics}

The baseline characteristics of the patients in both groups are shown in Table 1. The two groups were similar in age and sex distribution. There was no significant difference between the groups in Pathological staging and differentiation of adenocarcinoma (poorly/moderately/well by using 2010 World-Health-Organization criteria $[21])$.

\subsection{Intra-Operative Observation Indexes}

The Intra-operative observation indexes are shown in Table 2. In laparoscopic group, 12 patients were successfully performed assisted laparoscopic radical resection for mid transverse colon cancer, without conversion to laparotomy. Operation time was $200-300 \mathrm{~min}(230 \pm 28.60 \mathrm{~min})$, the bleeding volume was 50 $150 \mathrm{ml}(89.58 \pm 32.99 \mathrm{ml})$, intra-operative dissected lymph nodes number were 15 - 39 pieces $(22.25 \pm 6.48)$. In Open group, 13 patients were successfully 
Table 2. Comparison of operation time, intra-operative bleeding volume and the number of dissected lymph nodes in laparoscopic group with open group.

\begin{tabular}{cccc}
\hline Variable & Laparoscopic group $(\mathrm{n}=12)$ & Open group $(\mathrm{n}=13)$ & $P$ value \\
\hline operation time $(\mathrm{min})$ & $230.00 \pm 28.60$ & $213.85 \pm 33.55$ & 0.210 \\
bleeding volume $(\mathrm{ml})$ & $89.58 \pm 32.99$ & $133.46 \pm 44.51$ & 0.011 \\
number of lymph nodes $(\mathrm{n})$ & $22.25 \pm 6.48$ & $17.53 \pm 3.75$ & 0.035 \\
\hline
\end{tabular}

performed open radical resection for mid transverse colon cancer. Operation time was $160-270 \mathrm{~min}(213.85 \pm 33.55 \mathrm{~min})$, the bleeding volume was $70-200$ $\mathrm{ml}(133.46 \pm 44.51 \mathrm{ml})$, intra-operative dissected lymph nodes number were 13 25 pieces $(17.53 \pm 3.75)$. Operation time in laparoscopic group was longer than the open surgery group, but there was no significant difference in two groups $(\mathrm{p}>0.05)$. Intra-operative bleeding volume in laparoscopic group was obviously less than the open group $(\mathrm{p}<0.05)$. The number of dissected lymph nodes were more than open surgery group $(\mathrm{p}<0.05)$.

\subsection{Post-Operative Recovery Indexes}

The post-operative recovery indexes are shown in Table 3. Post-operative flatus time, liquid food intake time, ambulation time and hospitalization time in laparoscopic group were $2-5 \mathrm{~d}(3.00 \pm 1.12 \mathrm{~d}), 3-6 \mathrm{~d}(3.91 \pm 0.99 \mathrm{~d}), 1-6 \mathrm{~d}(3.33 \pm$ $1.43 \mathrm{~d})$ and $7-11 \mathrm{~d}(9.50 \pm 1.24 \mathrm{~d})$ respectively. Post-operative flatus time, liquid food intake time, ambulation time and hospitalization time in open group were $3-7 \mathrm{~d}(4.76 \pm 1.16 \mathrm{~d}), 4-8 \mathrm{~d}(5.00 \pm 1.15 \mathrm{~d}), 3-7 \mathrm{~d}(4.61 \pm 1.12 \mathrm{~d})$ and $10-16$ $\mathrm{d}(12.00 \pm 1.82 \mathrm{~d})$ respectively.

Index of post-operative recovery in laparoscopic group including post-operative flatus time, liquid food intake time, ambulation time and hospitalization time were shorter than the open group $(\mathrm{P}<0.05)$.

\subsection{Post-Operative Complications}

One case with post-operative incision site infection was found in open group. Abdominal bleeding, intra-abdominal infection, anastomotic fistula, intestinal obstruction weren't found in two groups. There wasn't re-hospitalization and dead case in $30 \mathrm{~d}$ post-operative period in two groups.

\subsection{Follow-Up Results}

Five patients (41.7\%) in laparoscopic group and six patients $(46.2 \%)$ in open group had died in 5-year follow-up. Figure 2 depicts the cumulative five-year overall survival curves, which were not significantly different between two groups $(\mathrm{P}=0.8547)$.

\section{Discussion}

After laparoscopic colon resection first reported in 1991 [1], laparoscopic surgery has been widely employed for various benign colorectal disease such as 


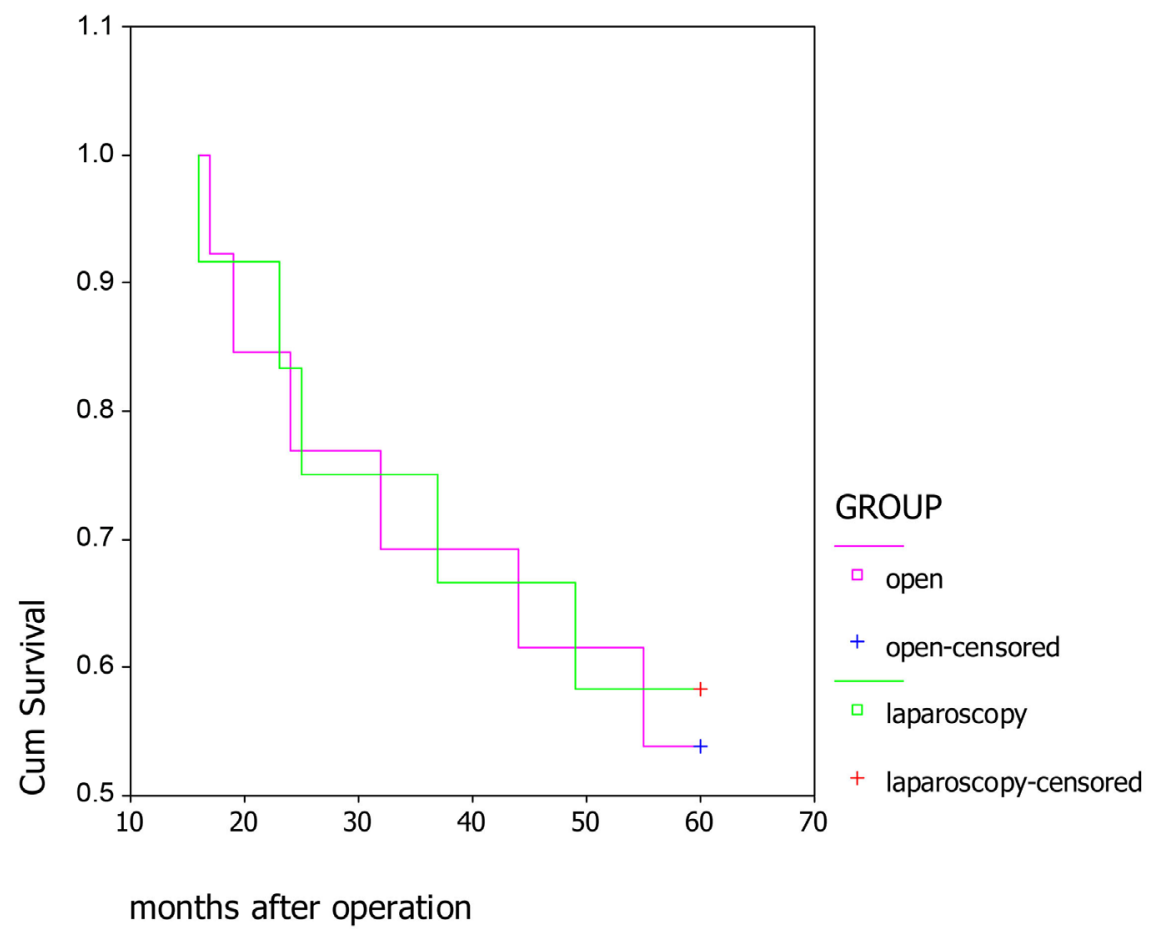

Figure 2. Kaplan-Meier curve comparing the cumulative five-year overall survival of patients of laparoscopic group $(n=12)$ and open group $(n=13)$.

Table 3. Comparison of post-operative flatus time, liquid food intake time, ambulation time and hospitalization time in laparoscopic group and open group.

\begin{tabular}{cccc}
\hline Variable & Laparoscopic group $(\mathrm{n}=12)$ & Open group $(\mathrm{n}=13)$ & $P$ value \\
\hline Flatus time (d) & $3.00 \pm 1.12$ & $4.76 \pm 1.16$ & 0.001 \\
liquid food intake time (d) & $3.91 \pm 0.99$ & $5.00 \pm 1.15$ & 0.020 \\
ambulation time (d) & $3.33 \pm 1.43$ & $4.61 \pm 1.12$ & 0.020 \\
hospitalization time (d) & $9.50 \pm 1.24$ & $12.00 \pm 1.82$ & 0.001 \\
\hline
\end{tabular}

benign mass [22], diverticular disease [23], inflammatory bowel disease [24], rectal prolapsed [25], and now increasingly for colorectal malignant disease [14] [15] [16]. Transverse colon cancer accounts for about $10 \%$ of cases of colorectal cancer, and the laparoscopic approach to oncological resection is a challenging task. Radical resection with laparoscopic assisted surgery for transverse colon cancer is difficult due to its special anatomical site, its proximity with adjacent structures such as pancreas, duodenum, spleen and the base of mesenteric vessels making it difficult for dissection and also the technical difficulties with laparoscopic identification, ligation, and lymph node dissection around the middle colic vessels. Because of these reasons, transverse colon cancer was excluded from almost every prior prospective randomized trial [11] [12] [13]. As experiences of laparoscopic surgery are accumulating and surgical techniques and instruments are developing, we consider that the extent of laparoscopic lymph node dissection for transverse colon cancer is not less than the extent of conventional lymph node dissection for transverse colon cancer. 
In our study from March 2006 to March 2014, we had analyzed 25 cases of patients with mid transverse colon cancer treatment. Laparoscopic assisted surgery for mid transverse colon cancer group had less intra operative bleeding, greater range of lymph node dissection as compared with open surgery group and the operation time in laparoscopic assisted surgery group was slightly longer than that with the open surgery group, but the difference was not statistically significant. The post-operative ambulation time, recovery of bowel function time, hospitalization time and the complication rates were significantly lesser in laparoscopic assisted surgery group as compared to the open group. Laparoscopic assisted transverse colon cancer surgeries are technically difficult, requiring facility with numerous surgical techniques and abilities to perform a diversity of procedures. For proximal transverse tumors or tumors at hepatic flexure, an extended right hemicolectomy is performed. Similarly, for distal transverse tumors, even at the splenic flexure, an extended left hemicolectomy is performed and for mid-transverse colon cancer transverse coloectomy is performed. In this study, through a retrospective analysis, we explore the skill of laparoscopic assisted radical resection of mid transverse colon cancer. During the laparoscopic assisted surgery for mid transverse colon cancer, firstly we followed the concept of Laparoscopic complete mesocolic excision (CME) with central vein ligation (CVL). The concept of complete excision of the organ within its primitive mesentery associated with central transection of the supplying blood vessels is today considered the step toward a modern surgical oncology, as the no-touch resection of the primitive embryological dorsal mesenterium is pivotal in achieving optimal local clearance. Furthermore, some studies suggested that CVL allows for an extensive lymph node clearance along the feeding vessels of the colon, with definitive effect on regional recurrence and systemic dissemination [26] [27]. Laparoscopic CME with CVL demonstrated not only superior oncologic efficacy but also to be absolutely safe, without increase in morbidity and mortality, with the addition of all the advantages of minimally invasive techniques [26]-[31]. Secondly, after resection of the mass the remaining bowel should be anastomosed for the continuity of bowel without any tension. It has been stated that "the key to a successful anastomosis is the accurate union of two viable bowel ends with complete avoidance of tension" [32]. Thus, the most important factors in the creation of a bowel anastomosis are: 1) meticulous technique; 2) good blood supply; and 3) no tension. During laparoscopic radical colon surgery, both hepatic flexure and splenic flexure of transverse colon are mobilized for a tension free anastomosis of remaining bowel. In our study: If the intestine was long enough, direct anastomosis of bowel without tension was done by end-to-end or end to side anastomosis. When colonic intestine is slightly tight after intestinal anastomosis, debonding of flexure duodenojejunalis can relieve the oppression to jejunum. There were 1 patients through debonding of flexure duodenojejunalis, relieve the oppression to jejunum, the prognosis is good. In the more obese patients or intestinal loop is thick and short bowel patients, in order to reduce the tension of anastomosis and the oppression to jejunum, pe- 
netration method (colon to be anastomosed through the small mesenteric root) is performed. The group also in 1 patient after the treatment, the prognosis is good. In addition, laparoscopic assisted transverse colon cancer operation, need surgery and assistants with skilled operation team, who have laparoscopic operation experience of the gastric, right and left colon cancer.

\section{Conclusion}

With good laparoscopic surgical skill, mid transverse colon cancer patient treated with laparoscopic assisted surgery has obvious advantages of the fine operation with curative resection and better clearance of lymph nodes. Laparoscopic assisted surgery group had less bleeding during the operation, the advantages of quick recovery after operation, without increasing the risk and complications.

\section{Conflicts of Interest}

The authors declare no conflicts of interest regarding the publication of this paper.

\section{References}

[1] Jacobs, M., Verdeja, J.C. and Goldstein, H.S. (1991) Minimally Invasive Colon Resection (Laparoscopic Colectomy). Surgical Laparoscopy \& Endoscopy, 1, 144-150.

[2] Law, W.L., Lee, Y.M., Choi, H.K., et al. (2007) Impact of Laparoscopic Resectiom for Colorectal Cancer on Operative Out-Comes and Survival. Annals of Surgery, 245, 1-7. https://doi.org/10.1097/01.sla.0000218170.41992.23

[3] Akmal, Y., Bailey, C., Baek, J.H., et al. (2011) Oncological Outcomes of Laparoscopic Colon Resection for Colon Cancer Implementation of a Full-Time Preceptorship. Surgical Endoscopy, 25, 2967-2971. https://doi.org/10.1007/s00464-011-1654-9

[4] Kuhry, E., Schwenk, W., Gaupset, R., et al. (2008) Long-Term Outcome of Laparoscopic Surgery for Colorectal Cancer: A Cochrane Systematic Review of Randomised Controlled Trials. Cancer Treatment Reviews, 34, 498-504. https://doi.org/10.1016/j.ctrv.2008.03.011

[5] Schwenk, W., Haase, O., Neudecker, J., et al. (2005) Short Term Benefits for Laparoscopic Colorectal Resection. Cochrane Database of Systematic Reviews, 20 , CD003145.

[6] Milsom, J.W., Bohm, B., Hammerhofer, K.A., et al. (1998) A Prospective Randomized Trial Comparing Laparoscopic versus C Conventional Techniques in Colorectal Cancer Surgery: A Preliminary Report. Journal of the American College of Surgeons, 187, 46-54. https://doi.org/10.1016/S1072-7515(98)00132-X

[7] Leung, K.L., Kwok, S.P., Lam, S.C., et al. (2004) Laparoscopic Resection of Rectosigmoid Carcinoma: Prospective Randomized Trial. Lancet, 363, 1187-1192. https://doi.org/10.1016/S0140-6736(04)15947-3

[8] Abraham, N.S., Byrne, C.M., Young, J.M., et al. (2007) Meta-Analysis of Non-Randomized Comparative Studies of the Short-Term Outcomes of Laparoscopic Resection for Colorectal Cancer. ANZ Journal of Surgery, 77, 508-516. https://doi.org/10.1111/j.1445-2197.2007.04141.x 
[9] Kahnamoui, K., Cadeddu, M., Farrokhyar, F., et al. (2007) Laparoscopic Surgery for Colon Cancer: A Systematic Review. Canadian Journal of Surgery, 50, 48-57.

[10] Tjandra, J.J. and Chan, M.K. (2006) Systematic Review on the Short-Term Outcome of Laparoscopic Resection for Colon and Rectosigmoid Cancer. Colorectal Disease, 8, 375-388. https://doi.org/10.1111/j.1463-1318.2006.00974.x

[11] Hazebroek, E.J. and Color Study Group (2002) COLOR: A Randomized Clinical Trial Comparing Laparoscopic and Open Resection for Colon Cancer. Surgical Endoscopy, 16, 949-953. https://doi.org/10.1007/s00464-001-8165-Z

[12] Clinical Outcomes of Surgical Therapy Study Group, Nelson, H., Sargent, D.J., et al. (2004) A Comparison of Laparoscopically Assisted and Open Colectomy for Colon Cancer. New England Journal of Medicine, 350, 2050-2059. https://doi.org/10.1056/NEJMoa032651

[13] Guillou, P.J., Quirke, P., Thorpe, H., et al. (2005) Short-Term Endpoints of Conventional versus Laparoscopic-Assisted Surgery in Patients with Colorectal Cancer (MRC CLASICC Trial): Multicentre, Randomised Controlled Trial. Lancet, 365, 1718-1726. https://doi.org/10.1016/S0140-6736(05)66545-2

[14] Cai, Y., Zhou, Y., Li, Z., et al. (2013) Surgical Outcome of Laparoscopic Colectomy for Colorectal Cancer in Obese Patients: A Comparative Study with Open Colectomy. Oncology Letters, 6, 1057-1062. https://doi.org/10.3892/ol.2013.1508

[15] Sun, J., Jiang, T., Qiu, Z., et al. (2011) Short-Term and Medium-Term Clinical Outcomes of laparoscopic-Assisted and Open Surgery for Colorectal Cancer: A Single Center Retrospective Case-Control Study. BMC Gastroenterology, 11, 85. https://doi.org/10.1186/1471-230X-11-85

[16] Mou, Y.P., Yang, P., Yan, J.F., et al. (2006) Clinical Evaluation of Laparoscopic Radical Resection of Colon Cancer. Chinese Journal of Surgery, 44, 581-583.

[17] West, N.P., Hohenberger, W., Weber, K., et al. (2010) Complete Mesocolic Excision with Central Vascular Ligation Produces an Oncologically Superior Specimen Compared with Standard Surgery for Carcinoma of the Colon. Journal of Clinical Oncology, 28, 272-278. https://doi.org/10.1200/JCO.2009.24.1448

[18] West, N.P., Kobayashi, H., Takahashi, K., et al. (2012) Understanding Optimal Colonic Cancer Surgery: Comparison of Japanese D3 Resection and European Complete Mesocolic Excision with Central Vascular Ligation. Journal of Clinical Oncology, 30, 1763-1769. https://doi.org/10.1200/JCO.2011.38.3992

[19] Aso, K. and Takahashi, T. (1988) Transverse Colon Resection. Gastroenterological Surgery, 11, 153-159.

[20] Toldt, C. (1919) Splanchology-General Considerations. In: Toldt, C. and Della Rossa, A., Eds., An Atlas of Human Anatomy for Students and Physicians, Vol. 4, Rebman Company, New York, 408.

[21] Hamilton, S., Bosman, F.T., Boffetta, P., et al. (2010) Carcinoma of the Colon and Rectum. In: Bosman, F.T.C.F., Hruban, R.H. and Theise, N.D., Eds., World Health Organization Classification of Tumours of the Digestive System, International Agency for Research on Cancer, Lyon, 134-146.

[22] Liska, D., Lee, S.W., Nandakumar, G., et al. (2012) Laparoscopic Surgery for Benign and Malignant Colorectal Diseases. Surgical Laparoscopy Endoscopy \& Percutaneous Techniques, 22, 165-174. https://doi.org/10.1097/SLE.0b013e31824be7ba

[23] Royds, J., O’Riordan, J.M., Eguare, E., et al. (2012) Laparoscopic Surgery for Complicated Diverticular Disease: A Single-Centre Experience. Colorectal Disease, 14, 1248-1254. https://doi.org/10.1111/j.1463-1318.2011.02924.x 
[24] Liu, C.D., Rolandelli, R., Ashley, S.W., Evans, B., Shin, M. and McFadden, D.W. (1995) Laparoscopic Surgery for Inflammatory Bowel Disease. The American Surgeon, 61, 1054-1056.

[25] de Hoog, D.E., Heemskerk, J., Nieman, F.H., et al. (2009) Recurrence and Functional Results after Open versus Conventional Laparoscopic versus Robot-Assisted Laparoscopic Rectopexy for Rectal Prolapse: A Case-Control Study. International Journal of Colorectal Disease, 24, 1201-1206. https://doi.org/10.1007/s00384-009-0766-3

[26] Le Voyer, T.E., Sigurdson, E.R., Hanlon, A.L., et al. (2003) Colon Cancer Survival Is Associated with Increasing Number of Lymph Nodes Analyzed: A Secondary Survey of Intergroup Trial INT-0089. Journal of Clinical Oncology, 21, 2912-2919. https://doi.org/10.1200/JCO.2003.05.062

[27] Chen, S.L. and Bilchik, A.J. (2006) More Extensive Nodal Dissection Improves Survival for Stages I to III of Colon Cancer: A Population-Based Study. Annals of Surgery, 244, 602-610.

[28] Lee, S.D. and Lim, S.B. (2009) D3 Lymphadenectomy Using a Medial to Lateral Approach for Curable Right-Sided Colon Cancer. International Journal of Colorectal Disease, 24, 295-300. https://doi.org/10.1007/s00384-008-0597-7

[29] Kanemitsu, Y., Komori, K., Kimura, K. and Kato, T. (2013) D3 Lymph Node Dissection in Right Hemicolectomy with a No-Touch Isolation Technique in Patients with Colon Cancer. Diseases of the Colon \& Rectum, 56, 815-824. https://doi.org/10.1097/DCR.0b013e3182919093

[30] West, N.P., Sutton, K.M., Ingeholm, P., et al. (2010) Improving the Quality of Colon Cancer Surgery through a Surgical Education Program. Diseases of the Colon \& Rectum, 53, 1594-1603. https://doi.org/10.1007/DCR.0b013e3181f433e3

[31] Bertelsen, C.A., Bols, B., Ingeholm, P., Jansen, J.E., Neuenschwander, A.U. and Vilandt, J. (2011) Can the Quality of Colonic Surgery Be Improved by Standardization of Surgical Technique with Complete Mesocolic Excision? Colorectal Disease, 13, 1123-1129. https://doi.org/10.1111/j.1463-1318.2010.02474.x

[32] Kirk, R.M. and Winslet, M. (2001) Essential General Surgical Operations. Churchill Livingstone, Edinburgh. 